\title{
Screening and analysis of pathogenic genes during DMBA- induced buccal mucosa carcinogenesis in golden hamsters
}

\author{
KAI YANG ${ }^{1}$, GUODONG ZHANG $^{1}$, JIE MEI $^{1}$, DAN CHEN $^{1}$ and MINGJUN WU ${ }^{2}$ \\ ${ }^{1}$ Department of Oral and Maxillofacial Surgery, the First Affiliated Hospital, Chongqing Medical University, Chongqing; \\ ${ }^{2}$ Institute of Life Sciences of Chongqing Medical University, Chongqing 400016, P.R. China
}

Received December 18, 2009; Accepted February 9, 2010

DOI: $10.3892 /$ or_00000803

\begin{abstract}
We designed to screen pathogenic genes related to the occurrence and development of oral buccal mucosa cancer by whole genome microarray and analyze the mechanisms of carcinogenesis. The golden hamster model of buccal mucosa cancer was established by induction with DMBA. cRNAs labeled with Cy3 were synthesized and hybridized with Agilent Whole Rat Genome Arrays containing 41,000 genes/ESTs. A Venn diagram analysis was performed to screen the continuously abnormally expressed genes. Our results show 5,255 significantly differentially expressed genes in golden hamster pouch mucosa during the progression of normal buccal mucosa to squamous cell carcinoma, of which 2,896 genes were up-regulated and 2,359 genes were down-regulated. Twenty-two genes were significantly differentially expressed at all stages of buccal mucosa carcinogenesis. Metabolism of xenobiotics by cytochrome P450 and the arachidonic acid metabolism pathway were closely related to each stage of buccal mucosa carcinogenesis. The expression changes of Eaf2, an up-regulated gene, and Ecg2, a down-regulated gene, determined by RT-PCR were consistent with the microarray. In conclusion, expression changes of various genes were involved in different stages of buccal mucosa carcinogenesis. The continuously abnormally expressed genes (Pthlh, Cyp2b13, Serpinb3, Cyp4b1, Ecg2, Btc, Krt10, Eaf2 and Trim2) at all stages of buccal mucosa carcinogenesis are important candidate genes for dynamic observation of the occurrence, development and prognosis of cancer. Searching for various effective inhibitors of xenobiotic metabolism by cytochrome P450 and the arachidonic acid metabolism pathway may suggest a therapy for the treatment and chemoprevention of oral squamous cell carcinoma.
\end{abstract}

Correspondence to: Dr Kai Yang, Department of Oral and Maxillofacial Surgery, the First Affiliated Hospital, Chongqing Medical University, Chongqing 400016, P.R. China E-mail: cqfyyk@hotmail.com

Key words: carcinogenesis test, computational biology, DNA microarrays, mucous membrane, oral buccal mucosa

\section{Introduction}

There are 274,000 new cases of oral cancer in the world every year (1). Squamous cell carcinoma accounts for $90 \%$ of oral cancer, and buccal carcinoma is one of the most common oral cancers (2). Although the treatment of oral cancers has markedly improved in recent decades, the 5-year survival rate for buccal carcinoma and other oral cancers is only 55$60 \%(2-4)$. Therefore, it is of great significance to explore the molecular mechanisms of the occurrence and development of oral mucosa carcinomas and search for effective therapeutic targets.

Current studies (5-7) demonstrate that oral mucosal carcinogenesis is a continuous dynamic process with multiple phases and steps. These changes occur due to expression changes in a number of genes. Different genes may play various roles at different stages of cancer development, but some genes may be continuously abnormally expressed at all stages of cancer development. At present, there are no reports of screening for such continuously abnor-mally expressed genes at all stages of the occurrence and development of oral mucosa (or buccal mucosa) cancer with high-throughput methods. However, it is of great importance to study these continuously expressed abnormal genes: First, changes in gene expression patterns at different stages of carcinogenesis reflect the specificity of the different stages of carcinogenesis, while continuously abnormally expressed genes at all stages of carcinogenesis represented the commonality of carcinogenesis during the whole development process. A comprehensive understanding of the specificity and commonality of the carcinogenesis process would aid in the exploration of further molecular mechanisms in the occurrence and development of buccal mucosa cancer. Second, a comprehensive understanding of the specificity and commonality of different carcinogenesis stages would contribute to the joint screening of effective therapeutic targets for these two aspects of buccal carcinoma. Previous studies also indicate that $80 \%$ of oral squamous cell carcinoma developed from precancerous lesions (8). In the clinic, precancerous lesions usually occur in various parts of the mouth of many patients. Some parts are cancerous, while others are still precancerous. Therefore, a comprehensive understanding of the specificity and commonality of different carcinogenesis stages would help with the selection of therapeutic targets, which not only play a role in the 
effective therapy of cancer foci, but also in preventing the development of precancerous lesions and the reversion of precancerous lesions. Last, a study of the continuously abnormally expressed genes at all stages of carcinogenesis would help in the screening of effective predictors of cancer occurrence, a dynamic observation of carcinogenesis and the prognosis for this type of cancer.

With the development of microarray technology, especially whole-genome microarrays in recent years, we can comprehensively understand genetic changes during carcinogenesis and screen related pathogenic genes at the global gene expression profile level. Currently, microarray technology has been applied to the study of oral tumor markers $(9,10)$, molecular typing of tumors $(11,12)$, drug screening $(5)$ and the comparison of gene expression profiles between cancer tissues and adjacent tissues or normal tissues (13-16). However, there are no reports of genome-wide screening of continuously abnormally expressed genes at all stages of the occurrence and development of oral mucosa (or buccal mucosa) cancer.

The experimental animal model of oral buccal squamous cell carcinoma is established by treating Syrian golden hamster buccal mucosa with DMBA (9,10-dimethylene-1,2benzanthracene) and was first reported by Salley in 1954 (17). Later studies $(18,19)$ demonstrated that the animal model not only simulated the pathological changes of human oral mucosal epithelial carcinogenesis but was also similar in growth characteristics and biological behavior. In addition, this animal model has many advantages, such as high stability, good repeatability and the superficial location of the tumor, which is easy to observe and cut. Therefore, this animal model was believed to be a good model for the study of buccal mucosa cancer.

In the present study, the Syrian golden hamster model of oral buccal squamous cell carcinoma was established by induction with DMBA. The Agilent Whole Rat Genome Array containing 41,000 genes/ESTs was used to detect the whole genome expression differences of normal buccal mucosa, precancerous lesions and squamous cell carcinoma. Combined with the bioinformatics analysis, the continuously abnormally expressed genes at all stages of the occurrence and development of buccal mucosa cancer were first screened, which provided a basis for further exploration of molecular mechanisms of the occurrence and development of oral mucosa cancer and the search for effective therapeutic targets.

\section{Materials and methods}

Main reagents and equipments. Syrian hamsters (Chengdu Institute of Biological Products, China), DMBA (Sigma, USA), a PCR thermocycler (PTC-100, MJ), an Agilent scanner (G2565AA, Agilent, USA), a spectrophotometer (ND1000, Nanodrop) and single-labeled Whole Rat Genome Arrays (Agilent) were used.

Methods. The establishment of animal models of buccal mucosa cancer. Fifteen male and fifteen female 6-8-weekold golden hamsters, weighing 90-120 g, were divided into groups I, II and III. There were 10 hamsters in each group. The hamsters were immobilized in a homemade rat frame, the cheek pouch was opened, and a $0.5 \%$ solution of DMBA in acetone was applied to bilateral cheek pouches with a No. 1 oil pen. The DMBA treatment was performed on Monday, Wednesday and Friday, and lasted for 12 weeks. All hamsters were subjected to food and drink deprivation for $2 \mathrm{~h}$ after DMBA treatment. The hamsters were sacrificed at day 0,6 weeks and 14 weeks after DMBA treatment, and their cheek pouches were removed. From each cheek pouch, half was immediately stored in liquid nitrogen, and the second half was fixed with $10 \%$ formalin, paraffin embedded, sliced and then stained with hematoxylin and eosin (HE). Pathological changes were observed using a light microscope. All experimental procedures were approved by the Laboratory Animal Management Committee of Experimental Animal Research Institute.

Extraction and purification of RNA from samples. RNA was extracted from samples of the three groups stored in liquid nitrogen with the Invitrogen TRIzol kit, and purified with the Qiagen RNeasy Kit according to the manufacturer's instructions. Equivalent RNA extracted from samples of the three groups was dissolved in Milli-Q water and stored at $-80^{\circ} \mathrm{C}$.

cRNA synthesis and labeling. The extracted RNA from samples of the three groups was single labeled with $\mathrm{Cy} 3$ by reverse transcription. The detailed steps were as follows: first, $2 \mu \mathrm{g}$ RNA and $5 \mu 1$ primers were added to a $1.5 \mathrm{ml}$ tube, and RNase-free water was added to make a total volume of $11.5 \mu \mathrm{l}$. Reactions were mixed well, placed in a $60^{\circ} \mathrm{C}$ water bath for $10 \mathrm{~min}$ and then in ice water to cool for $5 \mathrm{~min}$. Second, $2 \mu \mathrm{l}$ DDT at a concentration of $100 \mathrm{mmol} / \mathrm{l}$, $1 \mu 1$ dNTPs, $4 \mu 1$ buffer, $0.5 \mu 1$ RNase OUT and $1 \mu 1 \mathrm{MMLV}$ RT were added. Reactions were mixed well, centrifuged and heated to $40^{\circ} \mathrm{C}$ for $2 \mathrm{~h}$ with a $65^{\circ} \mathrm{C}$ thermal lid to synthesize cRNA. Third, the synthetic cRNA was labeled with aaUTP and purified with the Qiagen RNeasy Mini Kit. The concentration of the purified cRNA was measured using a spectrophotometer. Last, the cRNA was fluorescently labeled and re-purified.

Fragmentation of cRNA and chip hybridization. Cy3-labeled cRNA samples from the three groups at a quantity of $875 \mathrm{ng}$ were added to $11-\mu 1$ blocking agent, $2.2-\mu 1$ fragmentation buffer and nuclease-free water to a total volume of $55 \mu 1$. The cRNA mixtures were heated to $60^{\circ} \mathrm{C}$ and held for $30 \mathrm{~min}$ to be fragmented. The mixtures were then added to $55 \mu 12 \mathrm{X}$ GEX hybridization buffer and mixed. A volume of $100 \mu 1$ was added to the Whole Rat Genome Array and hybridized at $65^{\circ} \mathrm{C}$ and rolling at a speed of $10 \mathrm{rpm}$ for $17 \mathrm{~h}$. Then the chip was washed with washing buffer 1 for 1 min and then washing buffer 2 at $37^{\circ} \mathrm{C}$ for $1 \mathrm{~min}$.

Chip scanning and data analysis. The three chips were scanned with the Agilent G2565AA fluorescent scanner. The resolution of the scanner is $5 \mu \mathrm{m}$. The scanner automatically scanned the chips with 100 and 10\% PMT (Opto-coupled device). The two scanning results were merged by the Agilent software and normalized using Feature Extraction. In the present study, the normalized coefficient was 0.853 , and the ratio was higher than 3 , which is consistent with the experimental success criteria of the Agilent genome microarray. 
Table I. RT-PCR primers.

\begin{tabular}{lllc}
\hline Genes & \multicolumn{1}{c}{ Gene locus } & \multicolumn{1}{c}{ Sequences } & Length of PCR products (bp) \\
\hline Eaf2 & NM_172047 & F: 5'-AGCTGTCCCCAACGTCTCTA-3' & 165 \\
& & R: 5'-AGGATCGGTCATCATCTTCG-3' & 150 \\
Ecg2 & NM_001002816 & F: 5'-AAGCTCCTTGGTGGTCTCCT-3' & 130 \\
& & R: 5'-GATGTTTTCAATGGGGCAAG-3' & 130 \\
\hline
\end{tabular}
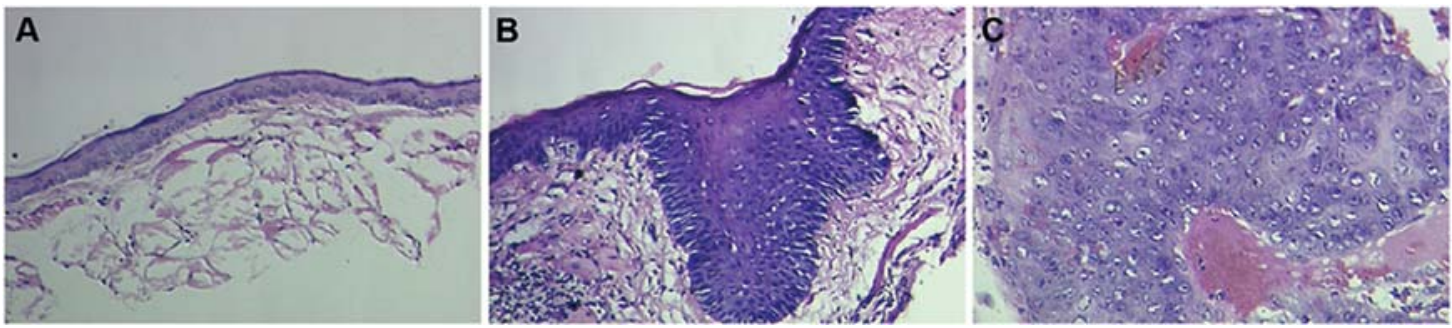

Figure 1. Pathological examination using a light microscope (x200). (A) Normal mucosa of golden hamster, (B) precancerous lesions of buccal mucosa (hyperplasia), (C) buccal mucosa squamous cell carcinoma.

The criteria for screening the differentially expressed genes were a ratio $\geq 2$ and a ratio $\leq 0.5$.

Bioinformatics analysis. The differentially expressed genes at different stages of carcinogenesis of buccal mucosa were analyzed with a Venn diagram with GeneSpring Software 10.0 (Agilent), and the continuously abnormally expressed genes at all stages of carcinogenesis were identified. The abnormally expressed genes were functionally classified using the classification criteria of Gene Ontology (http:// www.ncbi.nlm.nih.gov/). The signaling pathways involved with these genes were searched by analyzing the KECG, GENMAPP and BIOCARTA databases with the pathway analysis tools supplied by www.biorag.com. The significance of the signaling pathway was determined by $\mathrm{P}$-value $(\mathrm{P}<0.05)$, and the meaningful pathways were screened.

Validation of some differentially expressed genes by RT-PCR. The up-regulated gene Eaf2 and the down-regulated gene Ecg2 were randomly selected from the results of the Venn analysis and validated by RT-PCR. Total RNA was extracted from samples of all three groups according to the instructions of the TRIzol kit (Invitrogen). RNA at a quantity of $1 \mu \mathrm{g}$ was added to reverse transcriptase and random primers and was reverse transcribed into cDNA in a $20-\mu 1$ reaction according to the manufacturer's instructions. The PCR amplification reaction was performed as follows: the PCR program was started with pre-denaturation at $94^{\circ} \mathrm{C}$ for $5 \mathrm{~min}$, followed by 30 cycles of denaturation at $94^{\circ} \mathrm{C}$ for $15 \mathrm{sec}$, annealing at $55^{\circ} \mathrm{C}$ for $15 \mathrm{sec}$ and extension at $72^{\circ} \mathrm{C}$ for $20 \mathrm{sec}$, and ended with a final $72^{\circ} \mathrm{C}$ extension for $5 \mathrm{~min}$. PCR primers were designed using Primer 3.0 (Table I).

\section{Results}

Pathological examination at various stages of carcinogenesis. The results of the pathological examinations by conventional HE staining indicated that samples from groups I, II and III were normal buccal mucosa, precancerous lesions (hyperplasia) and squamous cell carcinoma, respectively (Fig. 1).

Screening of the significantly differentially expressed genes at various stages of carcinogenesis. There were 5,255 significantly differentially expressed genes in golden hamster buccal mucosa during the progression from normal buccal mucosa to precancerous lesions and then to squamous cell carcinoma. The distribution of significantly differentially expressed genes at various stages of carcinogenesis is shown in Table II.

\section{Bioinformatics analysis}

Venn diagram analysis. A Venn diagram analysis was used to analyze the distribution of differentially expressed genes among different expression chips based on the binomial distribution theory (Z-value). Venn diagram analysis can not only be used to analyze the distribution of differentially expressed genes in various groups of data from one chip but also analyze the distribution of differentially expressed genes in various groups of data from many chips. The distribution of 
Table II. The distribution of significantly differentially expressed genes at various stages of buccal mucosa carcinogenesis.

\begin{tabular}{lccc}
\hline Comparison between two groups & $\begin{array}{c}\text { Up-regulated } \\
\text { genes }\end{array}$ & $\begin{array}{c}\text { Down-regulated } \\
\text { genes }\end{array}$ & $\begin{array}{c}\text { Total differentially } \\
\text { expressed genes }\end{array}$ \\
\hline Precancerous lesions and normal mucosa & 747 & 584 & 1331 \\
Precancerous lesions and squamous cell carcinoma & 1362 & 619 & 1981 \\
Squamous cell carcinoma and normal mucosa & 787 & 1156 & 1943 \\
Total & 2896 & 2359 & 5255 \\
\hline
\end{tabular}

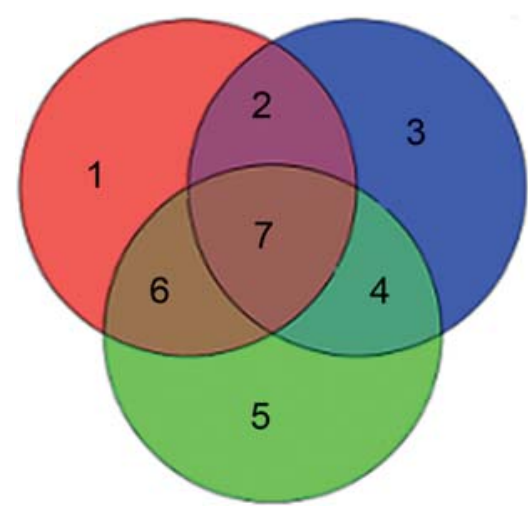

Figure 2. Venn diagram analysis of microarray results. Red circle, differentially expressed genes between precancerous lesions and buccal mucosa squamous cell carcinoma; blue circle, differentially expressed genes between normal buccal mucosa and precancerous lesions; green circle, differentially expressed genes between buccal mucosa squamous cell carcinoma and normal buccal mucosa.

Table III. The distribution of significantly differentially expressed genes in various sets by Venn diagram analysis.

\begin{tabular}{lcrc}
\hline Set & $\begin{array}{c}\text { Up-regulated } \\
\text { genes }\end{array}$ & $\begin{array}{c}\text { Down-regulated } \\
\text { genes }\end{array}$ & $\begin{array}{c}\text { Total differentially } \\
\text { expressed genes }\end{array}$ \\
\hline 1 & 196 & 434 & 630 \\
2 & 0 & 0 & 0 \\
3 & 225 & 156 & 381 \\
4 & 217 & 52 & 269 \\
5 & 314 & 281 & 595 \\
6 & 150 & 199 & 349 \\
7 & 4 & 18 & 22 \\
\hline
\end{tabular}

significantly differentially expressed genes at the two stages of buccal mucosa carcinogenesis analyzed by Venn diagram analysis is shown in Fig. 2 and Table III. Set 7 contained 22 genes (Table III) that were continuously abnormally expressed at all stages of buccal mucosa carcinogenesis.

Gene functional classification by gene ontology. Based on the GO functional classification criteria, the results of gene classification suggested that the 22 continuously abnormally expressed genes from Set 7 can be divided into seven major functional groups (Table IV).

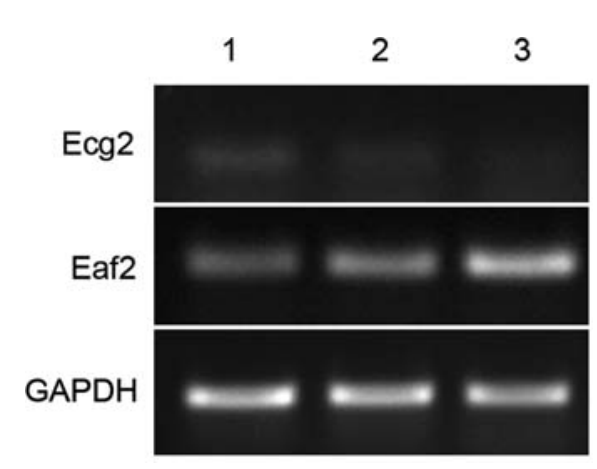

Figure 3. Results of RT-PCR. Lane 1, normal buccal mucosa; lane 2, precancerous lesions of buccal mucosa; lane 3 , buccal mucosa squamous cell carcinoma.

Pathway analysis. Through searching three major databases, two meaningful signaling pathways were found in the 22 significantly differentially expressed genes. Cyp2b13 was down-regulated in both pathways (Table V).

Validation of some differentially expressed genes by RT-PCR. Validation results of Eaf2, an up-regulated gene, and Ecg2, a down-regulated gene, are shown in Fig. 3. The optical density ratio was obtained by dividing the optical density value of PCR products of Eaf2 or Ecg2 with that of the internal standard, GAPDH (Table VI). The change tendency of the optical density ratio was consistent with the results of the microarray.

\section{Discussion}

Our study indicated that 5,255 genes were found significantly differentially expressed during the progression from normal buccal mucosa to precancerous lesions and then squamous cell carcinoma in golden hamsters, of which there were 2,896 up-regulated genes and 2,359 down-regulated genes. Our results demonstrated that buccal mucosa carcinogenesis was a complicated multi-stage pathological process and expression changes of various genes were involved. The aim of our study was to screen continuously abnormally expressed genes at all stages of buccal mucosa carcinogenesis. The results of the Venn diagram analysis and GO functional analysis showed that 22 genes were significantly differentially expressed at all stages of buccal mucosa carcinogenesis, of which there were 7 genes of unknown function $(31.8 \%)$, 6 metabolism-related genes $(27.3 \%)$, 3 signal transduction- 
Table IV. Functional classification of significantly differentially expressed genes in Set 7.

\begin{tabular}{lccl}
\hline Functional classification & Genes & Up-regulated genes & Down-regulated genes \\
\hline Metabolism-related genes & 6 & 0 & Ptpns113, Cyp2b13, Serpinb3, Cyp4b1, Ecg2, \\
Signal transduction-related genes & 3 & Pthlh & RGD1562284 \\
Cell structure-related genes & 3 & 0 & Kprp, Krt10, Ka24 \\
Transcriptional regulation-related genes & 1 & Eaf2 & 0 \\
Cell adhesion-related genes & 1 & 0 & Trim2 \\
Cell differentiation-related genes & 1 & Btc & 0 \\
Function unknown & 7 & AI454457 & AW143334, ENSRNOT00000001372, \\
& & & Tmem56, AA899770, TC617128, XM_227369 \\
\hline
\end{tabular}

Table V. Pathway analysis of differentially expressed genes at various stages of buccal mucosa.

\begin{tabular}{lcr}
\hline Pathway & P-value & Gene symbol \\
\hline Metabolism of xenobiotics by cytochrome P450 & 0.040 & Cyp2b13 \\
Arachidonic acid metabolism & 0.012 & Cyp2b13 \\
\hline
\end{tabular}

Table VI. Comparison between the results of RT-PCR and microarray.

\begin{tabular}{lcccc}
\hline Genes & Target gene mRNA $(\overline{\mathrm{x}} \pm \mathrm{s})$ & mRNA/GAPDH & A/B ratio & Gene array ratio \\
\hline & $\mathrm{A}$ & $\mathrm{B}$ & 4.83 & 5.54 \\
Eaf2 & $0.1592 \pm 0.0032$ & $0.7689 \pm 0.01542$ & -7.96 & -8.6 \\
Ecg2 & $0.3235 \pm 0.0043$ & $0.0406 \pm 0.0005$ & & \\
\hline
\end{tabular}

A, normal mucosa of golden hamster; B, precancerous lesions.

related genes $(13.6 \%), 3$ cell structure-related genes $(13.6 \%)$, 1 transcriptional regulation-related gene $(4.6 \%), 1$ adhesionrelated gene $(4.6 \%)$, and 1 cell differentiation-related gene $(4.6 \%)$. The results of the pathway analysis suggested that all stages of buccal mucosa carcinogenesis were closely related to the metabolism pathway of xenobiotics by cytochrome $\mathrm{P} 450$ and the arachidonic acid metabolism pathway.

Of the 22 significantly differentially expressed genes at all stages of buccal mucosa carcinogenesis, there were 15 genes with a known function. Previous studies (20-29) indicated that 9 of the 15 genes were associated with the occurrence and development of tumors or were abnormally expressed in tumor tissues. The up-regulation of the Pthlh gene was reported to promote cell proliferation (20). Ecg2 is a tumor suppressor gene whose down-regulation may promote cell proliferation and inhibit apoptosis (21). Serpinb3 was reported to be a member of the serine protease inhibitor family whose down-regulation may inhibit the apoptosis of tumor cells (22). Cyp2b13 and Cyp4b1 belong to the second and fourth cytochrome P450 family, respectively. They are involved in the metabolism of many carcinogens and toxins and may induce gene mutations and inhibit the expression of some genes (23-25). Btc was reported to be a member of the epidermal growth factor family, and its high expression may promote tumor angiogenesis (26). Krt10 is a marker of epithelial differentiation and its expression gradually decreased during malignant transformation of normal epithelial cells (27). Miyatake et al reported (28) that the expression of Trim2 was down-regulated in squamous cell carcinoma. Our results indicated that the expression pattern of the 8 genes above was the same as that reported by the previous studies. Xiao et al reported (29) that low expression and allelic loss of Eaf2 were found in prostate cancer, and Eaf2 deficiency may induce lung cancer, B cell lymphoma, liver cancer and prostate carcinoma. However, the expression of Eaf2 was up-regulated in the present study. This discrepancy may be due to different effects of Eaf2 in different tumors or other causes, which require further study. Cao et al reported (30) that an abnormal arachidonic acid metabolism pathway played an important role in the occurrence and development of oral cancer, which was consistent with our results. 
Although the present study was preliminary and further investigations on genetic polymorphisms, gene networks or protein functions were not conducted, our data combined with previous reports demonstrated that although various gene changes are involved in the different stages of carcinogenesis, the main mechanisms of DMBA-induced hamster buccal mucosa carcinogenesis are as follows: when DMBA, which is a chemical carcinogen with cytotoxicity and genotoxicity, was used to treat the oral buccal mucosa, the ability of cytochrome P450 and the arachidonic acid metabolism pathway to metabolize DMBA was decreased. Disorders of cell metabolism and the toxicity of DMBA may both induce expression changes of metabolism-related genes (such as Cyp2b13, Serpinb3, Cyp4b1 and Ecg2), signal transductionrelated genes (such as Pthlh), cell structure-related genes (such as Krt10) and cell differentiation-related genes (such as Btc), which promote cell proliferation and tumor angiogenesis and inhibit the apoptosis of tumor cells, resulting in the occurrence and development of cancer. Therefore, the continuously abnormally expressed genes (Pthlh, Cyp2b13, Serpinb3, Cyp4b1, Ecg2, Btc, Krt10, Eaf2 and Trim2) at all stages of buccal mucosa carcinogenesis are important candidate genes for dynamic observation of the occurrence, development and prognosis of buccal mucosa cancer. Searching for various effective inhibitors of the metabolism of xenobiotics by cytochrome P450 and the arachidonic acid metabolism pathway may suggest a therapy for the treatment and chemoprevention of oral squamous cell carcinoma.

\section{Acknowledgements}

The study was supported by Medical Foundation of Chongqing City Health Bureau (No. 03-2-073).

\section{References}

1. Parkin DM, Bray F, Ferlay J and Pisani P: Global cancer statistics, 2002. CA Cancer J Clin 55: 74-108, 2005.

2. Kademani D, Bell RB, Schmidt BL, Blanchaert R, Fernandes R, Lambert P and Tucker WM: Oral and maxillofacial surgeons treating oral cancer: a preliminary report from the American Association of Oral and Maxillofacial Surgeons Task Force on Oral Cancer. J Oral Maxillofac Surg 66: 2151-2157, 2008.

3. Bernier $\mathbf{J}$ and Cooper JS: Chemoradiation after surgery for high-risk head and neck cancer patients: how strong is the evidence? Oncologist 10: 215-224, 2005.

4. Kademani D, Bell RB, Bagheri S, Holmgren E, Dierks E, Potter B and Homer L: Prognostic factors in intraoral squamous cell carcinoma: the influence of histologic grade. J Oral Maxillofac Surg 63: 1599-1605, 2005.

5. Mendez E, Cheng C, Farwell DG, et al: Transcriptional expression profiles of oral squamous cell carcinomas. Cancer 95: 1482-1494, 2002.

6. Carinci F, Lo Muzio L, Piattelli A, et al: Genetic portrait of mild and severe lingual dysplasia. Oral Oncol 41: 365-374, 2005.

7. Choi $\mathrm{P}$ and Chen C: Genetic expression profiles and biologic pathway alterations in head and neck squamous cell carcinoma. Cancer 104: 1113-1128, 2005

8. Shiu MN and Chen TH: Impact of betel quid, tobacco and alcohol on three-stage disease natural history of oral leukoplakia and cancer: implication for prevention of oral cancer. Eur J Cancer Prev 13: 39-45, 2004

9. Nagata M, Fujita H, Ida $\mathrm{H}$, et al: Identification of potential biomarkers of lymph node metastasis in oral squamous cell carcinoma by cDNA microarray analysis. Int J Cancer 106: $683-689,2003$
10. Kashiwazaki H, Hassan NM, Hamada J, et al: Gene expression profile changes correlated with lymph node metastasis in oral squamous cell carcinoma. Odontology 96: 38-43, 2008.

11. Arora S, Matta A, Shukla NK, Deo SV and Ralhan R: Identification of differentially expressed genes in oral squamous cell carcinoma. Mol Carcinog 42: 97-108, 2005.

12. Erdem NF, Carlson ER and Gerard DA: Characterization of gene expression profiles of 3 different human oral squamous cell carcinoma cell lines with different invasion and metastatic capacities. J Oral Maxillofac Surg 66: 918-927, 2008.

13. Al Moustafa AE, Alaoui-Jamali MA, Batist G, et al: Identification of genes associated with head and neck carcinogenesis by cDNA microarray comparison between matched primary normal epithelial and squamous carcinoma cells. Oncogene 21: 2634-2640, 2002.

14. Gottschlich S, Ambrosch P, Cordes C, Gorogh T, Schreiber S and Hasler R: Gene expression profiling of head and neck squamous cell carcinoma using cDNA microarrays. Int J Oncol 29: 605-613, 2006.

15. Estilo CL, O-charoenrat P, Talbot S, et al: Oral tongue cancer gene expression profiling: identification of novel potential prognosticators by oligonucleotide microarray analysis. BMC Cancer 9: 11, 2009.

16. Tomioka H, Morita K, Hasegawa S and Omura K: Gene expression analysis by cDNA microarray in oral squamous cell carcinoma. J Oral Pathol Med 35: 206-211, 2006.

17. Salle JJ: Experimental carcinogenesis in the cheek pouch of the Syrian hanster. J Dermatol Res 33: 563, 1954.

18. Feng L and Wang Z: Chemopreventive effect of celecoxib in oral precancers and cancers. Laryngoscope 116: 1842-1845, 2006.

19. Gimenez-Conti IB and Slaga TJ: The hamster cheek pouch carcinogenesis model. J Cell Biochem (Suppl.) 17F: 83-90, 1993.

20. Hameetman L, Kok P, Eilers PH, Cleton-Jansen AM, Hogendoorn PC and Bovee JV: The use of Bcl-2 and PTHLH immunohistochemistry in the diagnosis of peripheral chondrosarcoma in a clinicopathological setting. Virchows Arch 446: 430-437, 2005.

21. Cui Y, Wang J, Zhang X, Lang R, Bi M, Guo L and Lu SH: ECRG2, a novel candidate of tumor suppressor gene in the esophageal carcinoma, interacts directly with metallothionein $2 \mathrm{~A}$ and links to apoptosis. Biochem Biophys Res Commun 302: 904-915, 2003.

22. Nakashima T, Yasumatsu R, Kuratomi Y, et al: Role of squamous cell carcinoma antigen 1 expression in the invasive potential of head and neck squamous cell carcinoma. Head Neck 28: 24-30, 2006

23. Wen X and Walle T: Preferential induction of CYP1B1 by benzo[a]pyrene in human oral epithelial cells: impact on DNA adduct formation and prevention by polyphenols. Carcinogenesis 26: 1774-1781, 2005.

24. Brookman-Amissah N, Mackay AG and Swann PF: Isolation and sequencing of the cDNA of a novel cytochrome P450 from rat oesophagus. Carcinogenesis 22: 155-160, 2001.

25. Sasaki T, Horikawa M, Orikasa K, et al: Possible relationship between the risk of Japanese bladder cancer cases and the CYP4B1 genotype. Jpn J Clin Oncol 38: 634-640, 2008.

26. Moon WS, Park HS, Yu KH, et al: Expression of betacellulin and epidermal growth factor receptor in hepatocellular carcinoma: implications for angiogenesis. Hum Pathol 37: 1324-1332, 2006.

27. Iwasa A, Oda Y, Kaneki E, et al: Squamous cell carcinoma arising in mature cystic teratoma of the ovary: an immunohistochemical analysis of its tumorigenesis. Histopathology 51: 98-104, 2007.

28. Miyatake T, Ueda Y, Nakashima R, et al: Down-regulation of insulin-like growth factor binding protein-5 (IGFBP-5): novel marker for cervical carcinogenesis. Int J Cancer 120: 2068-2077, 2007.

29. Xiao W, Zhang Q, Habermacher G, et al: U19/Eaf2 knockout causes lung adenocarcinoma, B-cell lymphoma, hepatocellular carcinoma and prostatic intraepithelial neoplasia. Oncogene 27: 1536-1544, 2008

30. Cao Y and Prescott SM: Many actions of cyclooxygenase-2 in cellular dynamics and in cancer. J Cell Physiol 190: 279-286, 2002. 